\title{
Factorized One-Way Wave Equations
}

\author{
Oskar Bschorr ${ }^{1}$ (D) and Hans-Joachim Raida ${ }^{2, *(D)}$ \\ 1 Aeroakustik Stuttgart, D-81679 Munich, Germany; o.bschorr@t-online.de \\ 2 Independent Researcher, D-42799 Leichlingen, Germany \\ * Correspondence: mail@hjraida.de; Tel.: +49-151-423-405-65
}

check for

updates

Citation: Bschorr, O.; Raida, H.-J. Factorized One-Way Wave Equations. Acoustics 2021, 3, 717-722. https:// doi.org/10.3390/acoustics3040045

Academic Editors: Xiongbing Li and Gaofeng Sha

Received: 22 October 2021

Accepted: 7 December 2021

Published: 9 December 2021

Publisher's Note: MDPI stays neutral with regard to jurisdictional claims in published maps and institutional affiliations.

Copyright: (c) 2021 by the authors. Licensee MDPI, Basel, Switzerland. This article is an open access article distributed under the terms and conditions of the Creative Commons Attribution (CC BY) license (https:// creativecommons.org/licenses/by/ $4.0 /)$.

\begin{abstract}
The method used to factorize the longitudinal wave equation has been known for many decades. Using this knowledge, the classical 2nd-order partial differential Equation (PDE) established by Cauchy has been split into two 1st-order PDEs, in alignment with D'Alemberts's theory, to create forward- and backward-traveling wave results. Therefore, the Cauchy equation has to be regarded as a two-way wave equation, whose inherent directional ambiguity leads to irregular phantom effects in the numerical finite element (FE) and finite difference (FD) calculations. For seismic applications, a huge number of methods have been developed to reduce these disturbances, but none of these attempts have prevailed to date. However, a priori factorization of the longitudinal wave equation for inhomogeneous media eliminates the above-mentioned ambiguity, and the resulting one-way equations provide the definition of the wave propagation direction by the geometric position of the transmitter and receiver.
\end{abstract}

Keywords: one-way wave equation; two-way wave equation; factorization; 1st-order partial differential equation; impulse flow equilibrium; inhomogeneous medium; bending wave; Moens-Korteweg wave; electromagnetic wave; Cauchy's first equation of motion

\section{Introduction}

Wave propagation in all three states of aggregation—solid, fluid and gas-is calculated according to Cauchy's equation of motion. For this 2nd-order partial differential Equation (PDE), two independent solutions exist and result in-according D'Alembert's separation approach-two wave equations representing two opposite traveling waves.

Unfortunately, D'Alembert's approach cannot be used to pre-select the task-relevant wave to identify the irrelevant wave. For analytical calculations, both solutions can be distinguished by their sign. However, in numerical finite element (FE) and finite difference (FD) calculations, the ambiguity causes irregular phantom effects known as "one-way/twoway wave" problem. Further related information with comprehensive lists of references is given in [1,2]. Several recently published patent applications [3-5] confirm that the problem still persists.

Factorization is a well-known method to solve reducible PDEs [6-10]. Specifically for homogeneous media, the second-order PDE wave equation can be factorized into two first-order PDEs, resulting in opposite traveling waves according to D'Alembert's solution. Generally, for wave propagation in homogeneous media, analytical solutions exist and calculation efforts for seconnd or first-order PDE do not differ significantly. However, in inhomogeneous media, waves can only be numerically calculated [11]. Seismic FE and FD calculations of geological structures require nodes in a Giga to Tera range and CPU computing times of days to weeks [12].

Thus, the primary task is the factorization of the wave equation for longitudinal waves in inhomogeneous media. Additionally, the factorization method for waves in homogeneous media is expanded to other wave types. 


\section{Method of PDE Factorization}

The homogeneous, isotropic and loss-free solid with a constant wave velocity $c[\mathrm{~m} / \mathrm{s}]$ serves as the starting point. Elastic deflection $s=s(x, t)[\mathrm{m}](t=$ time [seconds] $)$ is defined by Equation (1) and has two solutions (2), with waves traveling in $-x$ and $+x$ directions (angular frequency $\omega[\mathrm{rad} / \mathrm{s}]$, wave number $k=\omega / c[\mathrm{rad} / \mathrm{m}]$ )

$$
\left[\frac{\partial^{2}}{\partial t^{2}}-c^{2} \frac{\partial^{2}}{\partial x^{2}}\right] s=0 \quad(1) \quad \longrightarrow \quad s=s_{0} \sin (\omega t \pm k x)
$$

The ambiguity of the two solutions is problematic in the context of FE and FD calculations. Therefore, the well-known 2nd-order wave Equation (1) was repeatedly separated according to the expression $\left(a^{2}-b^{2}\right)=(a-b)(a+b)$ into two PDEs of the 1st order [13]:

$$
\left[\frac{\partial^{2}}{\partial t^{2}}-c^{2} \frac{\partial^{2}}{\partial x^{2}}\right] s=\left[\frac{\partial}{\partial t}-c \frac{\partial}{\partial x}\right]\left[\frac{\partial}{\partial t}+c \frac{\partial}{\partial x}\right] s=0
$$

In contrast with Equation (1), the resulting partial Equations (4) and (6) have the solutions (5) and (7) with the defined wave propagation direction $+c$ and $-c$, respectively.

$$
\begin{array}{lll}
{\left[\frac{\partial}{\partial t}-c \frac{\partial}{\partial x}\right] s=0} & (4) \quad \longrightarrow & s=s_{0} \sin (\omega t-k x) \\
{\left[\frac{\partial}{\partial t}+c \frac{\partial}{\partial x}\right] s=0} & (6) \quad \longrightarrow & s=s_{0} \sin (\omega t+k x)
\end{array}
$$

The factorization of a 2nd-order PDE into two independent first-order PDEs corresponds to the separation of a standing wave Equation (1) into two oppositely traveling wave Equations (4) and (6) and a change from force equilibrium to impulse equilibrium. Table 1 shows the solutions of Equation (1) and both first-order wave Equations (4) and (6).

Table 1. The 2nd-order (two-way) wave equation has two solutions. There are two standing waves, $\sin (\omega t) \cos (k x)$ and $\cos (\omega t) \sin (k x)$. According to $2 \sin (\alpha) \cos (\beta)=\sin (\alpha-\beta)+\sin (\alpha+\beta)$, two propagating waves $\sin (\omega t \pm k x)$ in opposite directions are the result, i.e., the solutions are ambiguous, and, after calculation, the appropriate solution has to be chosen. In contrast, the firstorder (one-way) wave equation has one propagating wave as the solution, with a pre-defined direction depending on the choice of the wave velocity $c=\{+c,-c\}$.

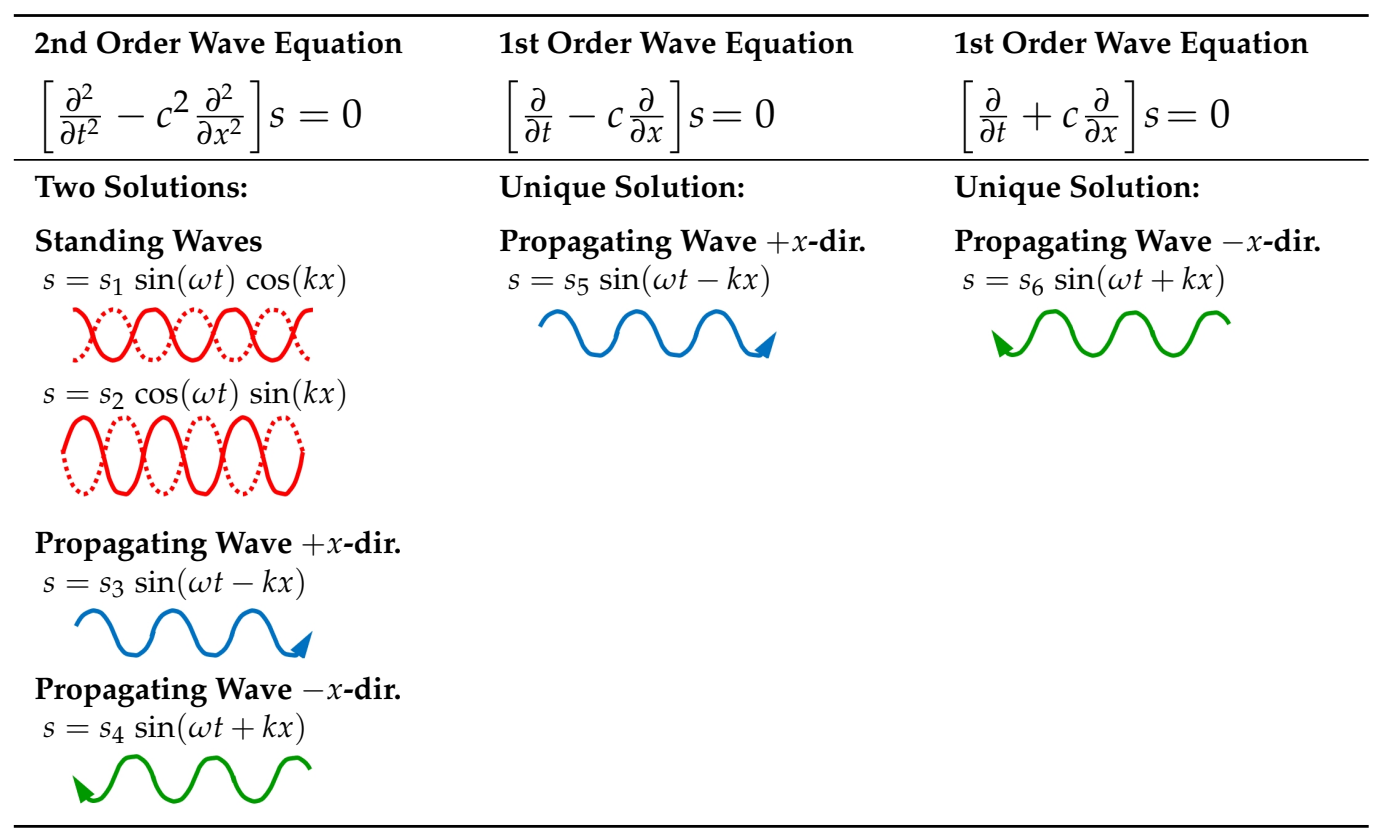




\section{Results}

\subsection{PDE Factorization - Waves in Inhomogeneous Media}

An inhomogeneous, isotropic medium with coordinates $x=\{x \boldsymbol{i}, y \boldsymbol{j}, z \boldsymbol{k}\}$ has a local density $\rho=\rho(\boldsymbol{x})\left[\mathrm{kg} / \mathrm{m}^{3}\right]$, a longitudinal elasticity modulus $E=E(\boldsymbol{x})[\mathrm{Pa}]$, and the wave velocity $c=c(x)=\sqrt{E / \rho}[\mathrm{m} / \mathrm{s}]$. The elastic displacement $s=\boldsymbol{s}(\boldsymbol{x}, t)[\mathrm{m}]$ is governed by the First Cauchy Equation of Motion:

$$
\rho \frac{\partial^{2}}{\partial t^{2}} s-\nabla^{2} E s=0
$$

To factorize this second-order PDE, $s$ is replaced by a new field variable Es. With the material law $\rho=E / c^{2}$ and $E \partial^{2} s / \partial t^{2}=\partial^{2} E s / \partial t^{2}$ (possible due to the time-invariant $E$ ), it follows that

$$
\frac{\partial^{2}}{\partial t^{2}}(E s)-c^{2} \nabla^{2}(E s)=\mathbf{0}
$$

Analogously to Equations (3), (4) and (6), this second-order wave equation for inhomogeneous media can be factorized into two first-order PDEs:

$$
\frac{\partial}{\partial t}(E s)-c \cdot \nabla(E s)=\mathbf{0} \quad(10) \quad \frac{\partial}{\partial t}(E s)+c \cdot \nabla(E s)=\mathbf{0}
$$

Longitudinal wave displacement $s$ and wave velocity $c$ have the same direction and can be turned in the direction of the $x \boldsymbol{i}$-axis $(s=s i, c=c i)$. Hence, the scalar form results:

$$
\frac{\partial}{\partial t}(E s)-c \frac{\partial}{\partial x}(E s)=0 \quad \text { with } \quad c=\{+c,-c\}
$$

The Helmholtz approach $\partial(E s) / \partial t=i \omega(E s)$ and settlement $(E s)^{\prime}=\partial(E s) / \partial x$ lead to

$$
i \omega(E s)-c(E s)^{\prime}=0 \quad(13) \quad \longrightarrow \quad(\ln E s)^{\prime}=\omega / c
$$

As can be controlled by insertion, the analytical solution (15) follows. Particle velocity $v[\mathrm{~m} / \mathrm{s}](16)$ and pressure $p[\mathrm{~Pa}](17)$ confirm impedance $z=|p / v|=\rho c$.

$$
\begin{gathered}
E s=E_{0} s_{0} \exp \left[i \omega\left(t-\int_{0}^{x} \frac{d x}{c}\right)\right] \\
v=\frac{\partial s}{\partial t}=(i \omega / E) E_{0} s_{0} \exp \left[i \omega\left(t-\int_{0}^{x} \frac{d x}{c}\right)\right] \\
p=\frac{\partial}{\partial x}(E s)=-(i \omega / c) E_{0} s_{0} \exp \left[i \omega\left(t-\int_{0}^{x} \frac{d x}{c}\right)\right]
\end{gathered}
$$

\subsection{PDE Factorization - Transversal Waves}

In case of homogeneity, a transversal wave with vectorial deflection $s$ and wave velocity $c$ is given by tensorial Equation (18). The insertion of $\Delta=\nabla \cdot \nabla=\nabla^{2}$ and successive factorization analogously to Equations (3), (4) and (6) yields one-way Equation (19)

$$
\ddot{s}-c^{2} \Delta s=0 \quad(18) \quad \longrightarrow \quad \dot{s}-c \cdot \nabla s=0
$$

A transversal wave consists of equal parts rotary and deviatoric portion, i.e.,

$$
\nabla s=(\nabla s)_{R o t}+(\nabla s)_{D e v}=2(\nabla s)_{R o t}=U \times \operatorname{rot} s
$$

Inserting this expression into Equation (19) and with $c \cdot U=c$, the transversal one-way wave Equation (21) follows as [14] (unit tensor $\boldsymbol{U}=\boldsymbol{i} \boldsymbol{i}+\boldsymbol{j} \boldsymbol{j}+\boldsymbol{k} \boldsymbol{k}$ )

$$
\dot{s}-c \times \operatorname{rot} s=0
$$




\subsection{PDE Factorization - Further Mechanical and Electromagnetic Waves}

According to PDE factorization (3) with the solutions for homogeneous media (4) and (6) further physical second- and fourth-order PDEs with the same structure as Equation (1) can also be factorized into first-order one-way wave equations in full analogy.

Table 2 lists classical equations for longitudinal, transversal, and string, Moens/ Korteweg, bending, and electromagnetic wave equations and their factorized pendants with the respective solutions. Additionally, governing formulas for wave velocity $c$ are given. The exclusion of asymmetrical tensor parts for mechanical waves and symmetrical tensor parts for electromagnetic waves are not carried over.

Table 2. Formal mathematical factorization of 2nd and 4th order partial differential wave equations into 1st order PDEs for homogeneous media. Wave-mechanically this corresponds to the separation of a standing wave into two complementary, in opposite directions travelling waves (here the wave propagating in $+x i$-direction is given). The electromagnetic longitudinal wave is an expansion of the longitudinal telegraph wave. Note: Wave propagation direction of the scalar factorized wave equations has to be defined by selection of the wave velocity $c=\{+c,-c\}$.

\begin{tabular}{|c|c|c|c|c|}
\hline Wave Type & $\begin{array}{l}\text { Conventional } \\
\text { Wave Equation } \\
=2 \text { nd Order PDE }\end{array}$ & $\begin{array}{l}\text { Factorized } \\
\text { Wave Equation } \\
=1 \text { st Order PDE }\end{array}$ & $\begin{array}{l}\text { Factorized Wave } \\
\text { Wave Direction +xi } \\
\text { Transversal dir. j,k }\end{array}$ & Wave Velocity \\
\hline Longitudinal Wave & $\ddot{s}-c^{2} \Delta s=0$ & $\dot{s}-c \cdot \nabla s=0$ & $s=i s_{0} \sin (\omega t-k x)$ & $c=\sqrt{E / \rho}$ \\
\hline Transversal Wave & $\ddot{s}-c^{2} \Delta s=\mathbf{0}$ & $\dot{s}-c \times \operatorname{rot} s=0$ & $s=j s_{0} \sin (\omega t-k x)$ & $c=\sqrt{G / \rho}$ \\
\hline Torsional Wave & $\ddot{\phi}-c^{2} \phi^{\prime \prime}=0$ & $\dot{\phi}-c \phi^{\prime}=0$ & $\phi=\phi_{0} \sin (\omega t-k x)$ & $c=\sqrt{G / \rho}$ \\
\hline String Wave & $\ddot{s}-c^{2} \Delta s=\mathbf{0}$ & $\dot{s}-c \times \operatorname{rot} s=0$ & $s=j s_{0} \sin (\omega t-k x)$ & $c=\sqrt{T / m}$ \\
\hline Moens/Korteweg Wave & $\ddot{\boldsymbol{s}}-c^{2} \boldsymbol{s}^{\prime \prime}=\mathbf{0}$ & $\dot{s}-c s^{\prime}=0$ & $s=i s_{0} \sin (\omega t-k x)$ & $c=\sqrt{E h / D \rho}$ \\
\hline Bending Wave & $m \ddot{\boldsymbol{s}}-\left(E J \boldsymbol{s}^{\prime \prime}\right)^{\prime \prime}=\mathbf{0}$ & $m c \dot{\boldsymbol{s}}-\left(E J \boldsymbol{s}^{\prime \prime}\right)^{\prime}=\mathbf{0}$ & $s=j s_{0} \sin (\omega t-k x)$ & $c=\sqrt[4]{\omega^{2} E J / m}$ \\
\hline Electromagnetic & $\ddot{E}-c^{2} \Delta E=\mathbf{0}$ & $\dot{E}-c \times \operatorname{rot} E=0$ & $E=j E s_{0} \sin (\omega t-k x)$ & $c=\sqrt{1 / \mu \epsilon}$ \\
\hline Transversal Wave & $\ddot{\boldsymbol{H}}-c^{2} \Delta \boldsymbol{H}=\mathbf{0}$ & $\dot{H}-c \times \operatorname{rot} H=0$ & $\boldsymbol{H}=\boldsymbol{k} H_{0} \cos (\omega t-k x)$ & $c=\sqrt{1 / \mu \epsilon}$ \\
\hline Longitudinal & $L C \ddot{U}-U^{\prime \prime}=0$ & $\dot{U}-c U^{\prime}=0$ & $U=i U_{0} \sin (\omega t-k x)$ & $c=\sqrt{1 / L C}$ \\
\hline Telegraph Wave & $L C \ddot{I}-I^{\prime \prime}=0$ & $\dot{I}-c I^{\prime}=0$ & $\boldsymbol{I}=i I_{0} \cos (\omega t-k x)$ & $c=\sqrt{1 / L C}$ \\
\hline Electromagnetic & $\ddot{E}-c^{2} \Delta E=\mathbf{0}$ & $\dot{E}-c \cdot \nabla E=0$ & $E=i E_{0} \sin (\omega t-k x)$ & $c=\sqrt{1 / \mu \epsilon}$ \\
\hline Longitudinal Wave & $\ddot{\boldsymbol{H}}-c^{2} \Delta \boldsymbol{H}=\mathbf{0}$ & $\dot{\boldsymbol{H}}-c \cdot \nabla \boldsymbol{H}=\mathbf{0}$ & $\boldsymbol{H}=\boldsymbol{i} H_{0} \cos (\omega t-k x)$ & $c=\sqrt{1 / \mu \epsilon}$ \\
\hline
\end{tabular}

Designation: $x \boldsymbol{i}, y j, z \boldsymbol{k}=$ cartesian coordinates; $s, s[\mathrm{~m}]=$ vectorial, scalar elastical displacement; $\dot{\boldsymbol{s}}=\partial \boldsymbol{s} / \partial t[\mathrm{~m} / \mathrm{s}]=$ particle velocity; $\ddot{s}=\partial^{2} s / \partial t^{2}\left[\mathrm{~m} / \mathrm{s}^{2}\right]=$ acceleration; ()$^{\prime}=\partial() / \partial x ; c=\{+c ;-c\}[\mathrm{m} / \mathrm{s}]=$ wave velocity; $\omega[\mathrm{rad} / \mathrm{s}]=$ angular frequency; $k[\mathrm{rad} / \mathrm{m}]=\mathrm{wave}$ number; ()$_{0}=$ amplitude; $E[\mathrm{~Pa}]=$ elasticity module; $G[\mathrm{~Pa}]=$ shear module; $\rho\left[\mathrm{kg} / \mathrm{m}^{3}\right]=$ density; $\phi\left[^{\circ}\right]=$ angle; $T[\mathrm{~N}]=$ tensile stress; $h[\mathrm{~m}]=$ duct wall thickness; $D[\mathrm{~m}]=$ duct diameter; $m[\mathrm{~kg} / \mathrm{m}]=$ mass distribution per meter; $E J\left[\mathrm{~Pa} \mathrm{~m}^{4}\right]=$ bending stiffness; $\boldsymbol{U}[\mathrm{V}]=$ electrical voltage; $I[\mathrm{~A}]=$ electric current; $E[\mathrm{~V} / \mathrm{m}]=$ electric field strength; $H[\mathrm{~A} / \mathrm{m}]=$ magnetic field strength; $C[\mathrm{As} / \mathrm{Vm}]=$ capacity per unit length; $L[\mathrm{Vs} / \mathrm{Am}]=$ inductivity per unit length; $\epsilon[\mathrm{As} / \mathrm{Vm}]=$ dielectricity constant; $\mu[\mathrm{Vs} / \mathrm{Am}]=$ permeability constant. 


\section{Discussion}

The main result is the factorization of the longitudinal wave equation for inhomogeneous media. Hereby, the elastic displacement $s=s(x, t)$ was replaced by the new field variable Es. Table 3 compares Cauchy's first Equation of Motion with the field variable $s$ and the factorized wave Equation (11) with the new field variable Es.

Table 3. Cauchy's 1st Equation of Motion (field variable: displacement $s[\mathrm{~m}]$ ) is based on force equilibrium, as can be seen from specific D'Alembert inertia force $\rho \ddot{s}\left[\mathrm{~N} / \mathrm{m}^{3}\right]=$ force per unit volume. In contrast, the one-way wave Equation (11) (new field variable: $E s$ ) resulting from the factorization of Cauchy's 1st Equation of Motion represents an impulse equilibrium and has the analytical solution (15).

\begin{tabular}{|c|c|c|c|c|c|}
\hline Equation & Concept & SI Units & PDE Type & Variable & Solutions for Inhomogeneity \\
\hline $\begin{array}{l}\text { Cauchy's 1st Eq. of Motion: } \\
\rho \frac{\partial^{2}}{\partial t^{2}} s-\Delta E s=\mathbf{0}\end{array}$ & $\begin{array}{l}\text { Force Equilibrium } \\
\text { Force Unit: } \mathrm{N}\end{array}$ & $\begin{array}{l}\mathrm{N} / \mathrm{m}^{3} \\
\mathrm{kgm} / \mathrm{s}^{2}\end{array}$ & $\begin{array}{l}\text { 2nd order } \\
\text { (Two-way) }\end{array}$ & $s$ & $\begin{array}{l}\text { Analytical solutions given for } \\
\text { some simple functions } E(x)\end{array}$ \\
\hline $\begin{array}{l}\text { Factorization: } \\
\frac{\partial}{\partial t}(E \boldsymbol{s})-\boldsymbol{c} \cdot \nabla(E \boldsymbol{s})=\mathbf{0}(11)\end{array}$ & $\begin{array}{l}\text { Impulse Equilibrium } \\
\text { Impulse Unit: Ns }\end{array}$ & $\begin{array}{l}\mathrm{N} / \mathrm{ms} \\
\mathrm{kgm} / \mathrm{s}\end{array}$ & $\begin{array}{l}\text { 1st order } \\
\text { (One-way) }\end{array}$ & Es & $\begin{array}{l}\text { General analytical solution: } \\
E s=E_{0} s_{0} \exp \left[i \omega\left(t-\int_{0}^{x} \frac{d x}{c}\right)\right](15)\end{array}$ \\
\hline
\end{tabular}

When inserting a constant elasticity module ( $E=$ const.) into factorized Equation (11), the one-way wave equation for homogeneous media results: $\dot{\boldsymbol{s}}-c \nabla s=0$, with the scalar form $\dot{s}-c s^{\prime}=0$. This wave equation obtained by (a) PDE factorization can also be derived from (b) the impedance theorem [15] and (c) the tensorial impulse flow equilibrium [16] of kinetic impulse flow $\rho c \dot{s}$ and potential impulse flow $E \nabla s$ (Table 4).

Table 4. Comparison of three different derivations of the one-way wave equation: (a) factorization of Cauchy's 1st Equation of Motion leads to longitudinal one-way wave equation in homogenous media and the travelling wave solution $s=s_{o} \sin (\omega t-k x)$. This result is confirmed by two further approaches: conversion of the (b) impedance theorem and (c) tensorial impulse flow equilibrium of kinetic impulse flow $\rho c \dot{s}$ and potential impulse flow $E \nabla s$. Analogous results follow for transversal waves in homogeneous media. The derivation of the one-way wave equation for longitudinal waves in inhomogenous media is achieved by PDE factorization and use of new field variable Es.

\begin{tabular}{lllll}
\hline $\begin{array}{l}\text { Mathematical/Physical } \\
\text { Approach }\end{array}$ & $\begin{array}{l}\text { Starting } \\
\text { Equation }\end{array}$ & $\begin{array}{l}\text { Conversion/ } \\
\text { Insertion }\end{array}$ & $\begin{array}{l}\text { Scalar One-Way } \\
\text { Wave Equation }\end{array}$ & $\begin{array}{l}\text { Traveling Wave } \\
\text { Solution }\end{array}$ \\
\hline (a) PDE Factorization & $\ddot{\boldsymbol{s}}-c^{2} \Delta \boldsymbol{s}=\mathbf{0}$ & $(\dot{\boldsymbol{s}}-\boldsymbol{c} \cdot \nabla \boldsymbol{s})(\dot{\boldsymbol{s}}+\boldsymbol{c} \cdot \nabla \boldsymbol{s})=\mathbf{0}$ & $\dot{\boldsymbol{s}}-c s^{\prime}=0$ & $s=s_{0} \sin (\omega t-k x)$ \\
(b) Impedance Theorem & $z=\rho c=p / v$ & $p=E s^{\prime} ; v=\dot{s} ; E=\rho c^{2}$ & $\dot{s}-c s^{\prime}=0$ & $s=s_{0} \sin (\omega t-k x)$ \\
(c) Impulse Equilibrium & $\rho c \dot{\boldsymbol{s}}-E \nabla \boldsymbol{s}=\mathbf{0}$ & $\boldsymbol{c} \cdot \boldsymbol{c}=c^{2} ; E=\rho c^{2} ; \dot{\boldsymbol{s}} \rightarrow \dot{s} ; \nabla s \rightarrow s^{\prime}$ & $\dot{s}-c s^{\prime}=0$ & $s=s_{0} \sin (\omega t-k x)$ \\
\hline
\end{tabular}

\section{Conclusions}

The classical approach of calculating wave propagation via 2nd order PDE or "Twoway wave equation" is standardely used, although it primarily describes a standing wave field and ambiguous solutions result. If the calculation of wave propagation only in a given direction is of interest, such in seismics, the 1st order PDE or "One-way wave equation" is necessary. Because there exists no analytical solution for the one-way wave equation in inhomogeneous media approximations have to be used. However, this gap has been closed by the analytical solution presented in this article. Moreover, the method of factorization can be analogously tranferred to other standard wave forms and electromagnetic waves. This creates opportunities for simpler and faster wave propagation calculations in this field of research and also in other technical areas. 
Author Contributions: Conceptualization; methodology; formal analysis; O.B.; writing-original draft preparation; O.B. and H.-J.R.; investigation; H.-J.R.; writing-review \& editing; O.B. and H.-J.R.; visualization; H.-J.R. All authors have read and agreed to the published version of the manuscript.

Funding: This research received no external funding.

Data Availability Statement: Not applicable.

Conflicts of Interest: The authors declare no conflict of interest.

\section{References}

1. Angus, D.A. The One-Way Wave Equation: A Full-Waveform Tool for Modeling Seismic Body Wave Phenomena. Surv. Geophys. 2014, 35, 359-393. [CrossRef] [CrossRef]

2. Luo, M.; Jin, S. Halliburton Energy Services: Hybrid One-Way and Full-Way Wave Equation Migration. U.S. Patent Nr. 8116168B1, 14 February 2012.

3. Yan, J.; Anderson, J.E.; Lu, R.; Marcinkovich, C.M. Exxonmobile Upstream Research Company. Efficient Seismic Attribute Gather Generation With Data Synthesis Furthermore, Expectation Method. WO Patent Nr. 2017136133A1, 10 August 2017.

4. Zhou, M. China Petrol \& Chemical Corporation: Method and Device for Enhanced Seismic Imaging Based on One-Way Wave Equation. U.S. Patent Nr. 10962669B2, 30 March 2021.

5. Bai, J.; Yilmaz, O. Emerson Paradigm Holding LLC. Seismic Migration Techniques for Improved Image Accuracy. U.S. Patent Nr. 2021215824A1, 15 July 2021.

6. Schlesinger, L. Handbuch der Theorie der Linearen Differentialgleichungen; Teubner Verlag: Leipzig, Germany, 1897.

7. Kamke, E. Differentialgleichungen: Lösungsmethoden und Lösungen; Akademische Verlagsgesellschaft: Leipzig, Germany, 1965.

8. Davis, J.L. Mathematics of Wave Propagation; Princeton University Press: Princeton, NJ, USA, 2000; p. 85.

9. Garrett, S.L. Understanding Acoustics; An Experimentalist's View of Acoustics and Vibration; Springer: Berlin, Germany, 2017 ; p. 136.

10. Olver, P.J. Introduction to Partial Differential Equations; Springer: Berlin, Germany, 2016; p. 51.

11. Elsevier, B.V. Wave Propagation in Inhomogeneous Media. Int. Geophys. 1972, 17, 223-307. [CrossRef]

12. Seriani, G.; Oliveira, S.P. Numerical modeling of mechanical wave propagation. Riv. Nuovo C 2020, 43, 460. [CrossRef] [CrossRef]

13. Lamoureux, M.P. The Mathematics of PDEs and the Wave Equation; Seismic Imaging Summer School, University of Calgary: Calgary, AB, Canada, 2006. [CrossRef]

14. Bschorr, O.; Raida, H.-J. Transversal One-Way Wave Equation. In Proceedings of the DAGA, Hannover, Germany, 16-19 March 2020; pp.1075-1076.

15. Bschorr, O.; Raida, H.-J. One-Way Wave Equation Derived from Impedance Theorem. Acoustics 2020, 2, 12. [CrossRef] [CrossRef]

16. Bschorr, O.; Raida, H.-J. Spherical One-Way Wave Equation. Acoustics 2021, 3, 21. [CrossRef] [CrossRef] 